\title{
Novel Robust Walking for Biped Robot Using Adaptive Neural PID Controller
}

\author{
Ho Pham Huy Anh ${ }^{1} \quad$ Tran Thien Huan ${ }^{2} \quad$ Nguyen Thanh Nam ${ }^{3}$ \\ ${ }^{1}$ Corresponding author, FEEE, Ho Chi Minh City University of Technology, Ho Chi Minh City, Viet Nam \\ (Tel: +84-908229736; Email: hphanh@ @cmut.edu.vn) \\ 1, 2, 3 DSCELAB, Viet Nam National University Ho Chi Minh City (VNU-HCM), Viet Nam
}

\begin{abstract}
This paper presents the design, development and implementation of a novel adaptive neural PID (AN-PID) controller suitable for real-time robust walking biped robot control application. The unique feature of the proposed $\mathrm{AN}$ PID controller is that it has highly simple and dynamic selforganizing structure, fast online-tuning speed and flexibility in online-updating. The proposed adaptive algorithm focuses on efficiently optimizing Gain Scheduling and PID weighting parameters of neural MLPNN model integrated in the proposed AN-PID controller. This implemented AN-PID controller aims to successfully control the robust walking of the highly nonlinear full-sized biped robot HUBOT-3. The performance of this novel neural-based PID controller was found to be outperforming in comparison with conventional PID controller.
\end{abstract}

Keywords: Biped robot; Adaptive Neural PID (AN-PID) controller; Auto-Tuning Gain Scheduling; Robust Walking

\section{INTRODUCTION}

Walking biped robots could be classified in three different categories. The first category represents static walkers, whose motion is very slow such that system is stability is completely described. The projected center of mass is constantly inside the supporting polygon area. The second category represents dynamic walkers, biped robots having feet and actuated ankles. The Zero Moment Point (ZMP) [1] is situated in the supporting area. These walkers are potentially able to move in a static way, provided that they have large enough feet and motion is slow. Third category represents purely dynamic walking robots without having feet. With criteria of dynamic balance, the projected center of mass is allowed outside of the area inscribed by the feet and the walker may essentially fall during walking gait cycles.

The control problem for dynamic walking is more complicated than walking with static balance. Dynamic walking patterns provide higher walking speed and efficiency with more versatile walking structures. Among all mentioned categories of walking robots, the issue of stable and reliable biped walk is the most fundamental and yet unsolved with a high degree of reliability. The question has motivated formation of several dynamic based criteria for the evaluation and control of balance in biped locomotion. However, the ZMP is used as stabilizing index in this paper.

Several methodologies have been developed for the control of biped walking robots, from model based modern control [2-4], to various intelligent control systems [5-8].
However, it is still difficult to maintain stable and smooth locomotion while the biped is dynamic walking.

As one of methodologies applied for biped gait synthesis and control of biped and humanoid robot, some researchers used fuzzy logic. Fuzzy logic was used dominantly as parts of control systems on executive control level, for generating and tuning PID gains [5-6]. Both fuzzy and neuro control are model-free design methods. However, because multiDOFs biped robot is a multivariable, high-order, strongcouple nonlinear dynamic system, it is difficult to get fuzzy control rules by using human expert-based or supervised neural network by using numerical data methods [7-8].

This paper proposes an adaptive neural AN-PID controller that possesses good features such as highly simple and dynamic self-organizing structure, fast learning speed, good generalization and flexibility in learning. The proposed online tuning AN-PID controller is employed to compensate for environmental variations such as payload mass and timevarying parameters during the operation process. By virtue of on-line training by back propagation (BP) learning algorithm and then auto-tuned gain scheduling $K$ and PID weighting values $K_{p}, K_{i}$ and $K_{d}$, it learns well the nonlinear biped robot dynamics and simultaneously makes control decisions to both of legs of the biped robot as to ensure a stable and robust walking.

The rest of this paper is organized as follows. The section 2 introduces the mechanical and electrical structure of biped robot HUBOT-3. The section 3 presents procedure of design an online tuning gain scheduling AN-PID controller for the robust walking of a full-sized biped robot. The section 4 presents and analyses experiment studies and results. Finally, the conclusion belongs to the section 5 .

\section{EXPERIMENTAL DESCRIPTION}

The photograph of the full-sized biped robot (HUBOT-3) and its structure are illustrated in Fig. 1. Its total weight, including computer, controllers and amplifiers, is $50 \mathrm{~kg}$ and its height is about $140 \mathrm{~cm}$. The full-sized biped robot has 24 d.o.f.. Each leg has 6 DOF and it can imitate human walking motion in both of sagittal and frontal planes. The full-sized biped robot (HUBOT-3) was designed to have a kinematically adequate structure. A complicated mechanical design such as a differential mechanism was avoided. Harmonic drive gears were used as the main reduction gears. 


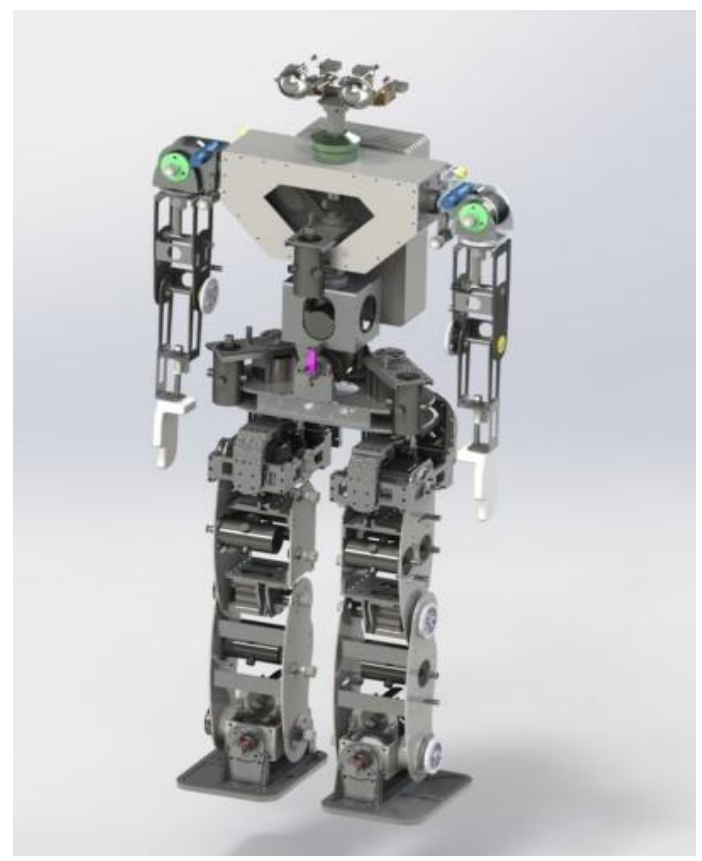

Fig. 1 Photograph of the full-sized biped robot HUBOT-3

Actuators of the lower limbs were selected by the simulation of specific motion patterns in the sagittal and lateral planes with the $3 \mathrm{D}$ simulation environments V-REP designed and implemented for biped robot testing. In this study, we focus on sensory feedback using potentiometers,
IMU and F/T sensors. After maximizing the performance with adequate sensors, we enhance sensory limits with additional sensors in the future research later on. The ZMP is simply the center of the pressure of the foot on the ground, and the moment applied by the ground about this point is zero. In other words, it is a point $\mathrm{P}$ on the ground at which the net moment of the inertial and gravity forces has no component along the axes parallel to the ground. Thus, it is necessary to measure the F/T of the foot in order to calculate the actual ZMP. The biped robot is equipped with eight flexi-force sensors that are integrated in both feet.

\section{CONTROLLER DESIGN}

The structure of the newly proposed online tuning adaptive neural AN-PID control algorithm based on neural network is represented in Fig. 2. This control algorithm is a new one and possesses the very good features such as simple structure and little computation time, compared with the previous neural network controller using auto-tuning method [15] (2005). This system with the set point filter and controller using neural network can solve the problems, which were mentioned in the introduction and proves very useful for the robust walking control of the highly nonlinear full-sized biped robot.

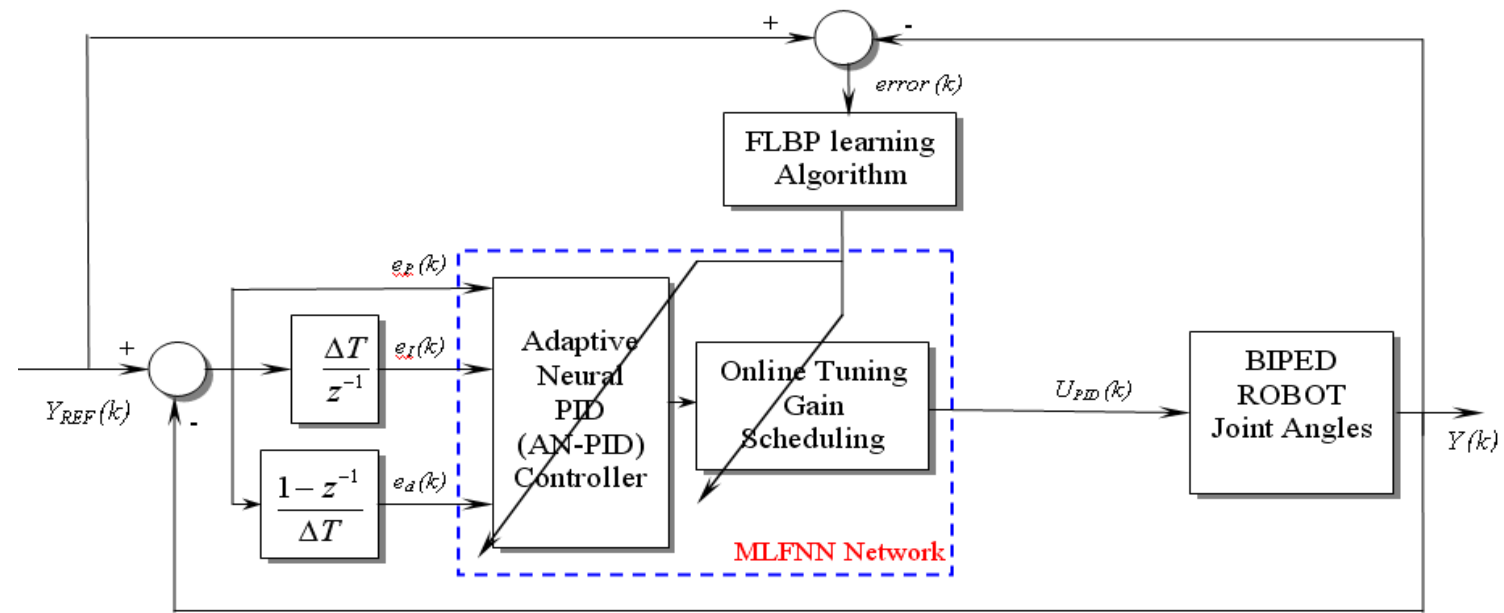

Fig.2 Block diagram of proposed online tuning adaptive neural AN-PID controller for biped robot's position control system

The structure of the newly proposed online tuning adaptive neural AN-PID control algorithm using MultiLayer Feed-forward Neural Network (MLFNN) is illustrated in Fig.3. This control algorithm is a new one and possesses the characteristics such as simple structure, little computation time and more robust control, compared with the previous neural network controller using auto-tuning method [9].

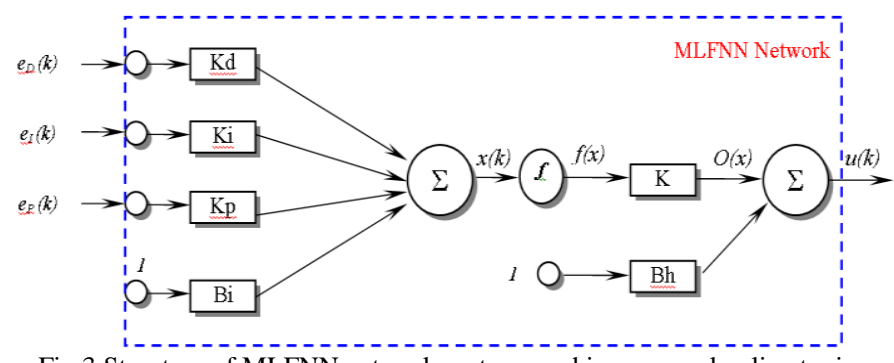

Fig.3 Structure of MLFNN network system used in proposed online tuning adaptive neural AN-PID controller 
From Figs. 2 and 3, a control input $u$ applied to the joint angles' DC motor drive of the full-sized biped robot can be obtained from the following equation.

$u=K f(x)+B_{h}$

with $x$ is input of Hyperbolic Tangent function $f($.) which is presented in Equation (2), $K$ and $B_{h}$ are the bias weighting values of input layer and hidden layer respectively. The Hyperbolic Tangent function $f($.) has a nonlinear relationship as explained in the following equation.

$$
f(x)=\frac{\left(1-e^{-x}\right)}{\left(1+e^{-x}\right)}
$$

In Fig. 3, $K, K_{p}, K_{i}$ and $K_{d}$, are scheduling, proportional, integral and derivative gain while $e_{p}, e_{i}$ and $e_{d}$ are system error between desired set-point output and output of joint angle of the full-sized biped robot, integral of the system error and the difference of the system error, respectively.

MLFNN network is trained online by the fast learning back propagation (FLBP) algorithm as to minimize the system error between desired set-point output and output of joint of the PAM robot arm.

From Fig. 3, the input signal of the Hyperbolic Tangent function $f($.) becomes

$$
\begin{aligned}
& x(k)=K_{p}(k) e_{p}(k)+K_{i}(k) e_{i}(k)+K_{d}(k) e_{d}(k)+B_{i}(k) \\
& O(k)=f(x(k)) \\
& u(k)=K(k) O(k)+B_{h}(k) \\
& \text { with } \\
& e_{p}(k)=y_{R E F}(k)-y(k) \\
& e_{i}(k)=e_{p}(k) \cdot \Delta T \\
& e_{d}(k)=\frac{e_{p}(k)\left(1-z^{-1}\right)}{\Delta T} \\
& K(k+1)=K(k)-\eta \frac{\partial E(k)}{\partial K} \\
& K_{p}(k+1)=K_{p}(k)-\eta_{p} \frac{\partial E(k)}{\partial K_{p}} \\
& K_{i}(k+1)=K_{i}(k)-\eta_{i} \frac{\partial E(k)}{\partial K_{i}} \\
& K_{d}(k+1)=K_{d}(k)-\eta_{d} \frac{\partial E(k)}{\partial K_{d}}
\end{aligned}
$$

where $\Delta T$ is the sampling time, $z$ is the operator of $Z$ Transform, $k$ is the discrete sequence, $y_{R E F}(k)$ and $y(k)$ are the desired set-point output and output of joint of the PAM robot arm. Furthermore, $B_{i}, K_{p}, K_{i}$ and $K_{d}$ are weighting values of Input layer and $B_{h}$ and $K$ are weighting values of Hidden layer. These weighting values will be tuned online by fast learning back propagation (FLBP) algorithm.

As to online tuning the gain scheduling $K$ and PID parameters $K_{p}, K_{i}$ and $K_{d}$, the gradient descent method used in FLBP learning algorithm using the following equations were applied. Furthermore, The Bias weighting values $B_{i}(k)$ and $B_{h}(k)$ are updated as follows:
$B_{i}(k+1)=B_{i}(k)-\eta_{B i} \frac{\partial E(k)}{\partial B_{i}}$

$B_{h}(k+1)=B_{h}(k)-\eta_{B h} \frac{\partial E(k)}{\partial B_{h}}$

where $\eta, \eta_{p}, \eta_{i}, \eta_{d}, \eta_{B i}$ and $\eta_{B h}$ are learning rate values; $\mathrm{E}(\mathrm{k})$ is the error defined by the gradient descent method. From (5)-(6), the final equations for online tuning gain scheduling $K$ and $K_{p}, K_{i}$ and $K_{d}$ are expressed as follows:

$K(k+1)=K(k)+\eta \cdot e_{p}(k) \Delta \cdot O(k)$

$K_{p}(k+1)=K_{p}(k)+\eta_{p} \cdot e_{p}^{2}(k) \Delta K \cdot \frac{2 e^{-x}}{\left(1+e^{-x}\right)^{2}}$

$K_{i}(k+1)=K_{i}(k)+\eta_{i} \cdot e_{p}(k) e_{i}(k) \Delta K \frac{2 e^{-x}}{\left(1+e^{-x}\right)^{2}}$

$K_{d}(k+1)=K_{d}(k)+\eta_{d} \cdot e_{p}(k) e_{i}(k) \Delta K \frac{2 e^{-x}}{\left(1+e^{-x}\right)^{2}}$

Finally, the Bias weighting values $B_{i}(k)$ and $B_{h}(k)$ are updated as follows:

$B_{i}(k+1)=B_{i}(k)+\eta_{B i} \cdot e_{p}(k) \Delta K \frac{2 e^{-x}}{\left(1+e^{-x}\right)^{2}}$
$B_{h}(k+1)=B_{h}(k)+\eta_{B h} e_{p}(k) \Delta$

\section{EXPERIMENTAL RESULTS}

The performance of proposed online tuning gain scheduling AN-PID control scheme is verified on joint angle position control of the full-sized biped robot. Fig.2 and Fig.3 describes the working diagram of this control scheme with the experiment SIMULINK diagram of proposed online tuning AN-PID control algorithm run in Real-time Windows Target.

The final purpose of the biped robot joint angle trajectory tracking is to keep a robust walking for 12 joint angles of both left and right legs. Thus, the experiments were carried out with respect to 3 different waveforms as reference input (Triangular, Trapezoidal and Sinusoidal reference) with 2 left and right legs' joint angles of biped robot as to demonstrate the performance of novel proposed online tuning AN-PID controller. Furthermore, the comparisons of control performance between the conventional PID and two different methods of the proposed online tuning AN-PID controller were performed.

These two novel proposed methods compose of proposed online tuning AN-PID-SIG and proposed online tuning AN-PID-HYP. The $1^{\text {st }}$ method possesses the activation function of hidden layer of AN_PID subsystem being Sigmoid function and the $2^{\text {nd }}$ method corresponds to the Hyperbolic Tangent function respectively. The initial gain scheduling value $G$ and PID controller parameters $K_{p}$, $K_{i}$ and $K_{d}$ were set to be $G=0.8, K p=0.089, K i=0.09, K d$ $=0.07$. These parameters of PID controller were obtained by GA optimization method. 
The experiments were carried out to verify the effectiveness of the proposed AN-PID controller with trapezoidal reference input. Fig. 6 shows the experimental results in comparison between the conventional PID controller and the two proposed nonlinear AN-PID-SIG and AN-PID-HYP controllers in 2 left and right joint angles of biped respectively. The online updating of each control parameter $(G, K p, K i$ and $K d$ ) of 2 left and right joint angles was shown in Fig. 7. In the experiment of the proposed online tuning AN-PID controller, the initial values of $G, K p$, $K i$ and $K d$ are set to be the same as that of PID controller.

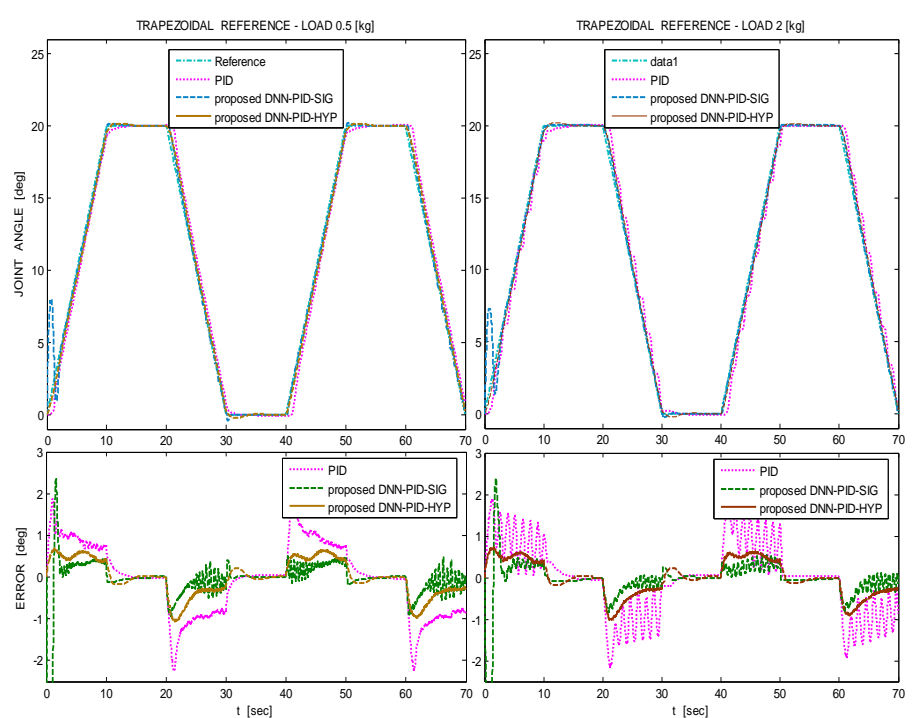

Fig.6 Trapezoidal response of two joints (belong to left and right leg) of the biped robot

These figures show that thanks to the refined online tuning of $G, K p, K i$ and $K d$, the error between desired reference $y_{R E F}$ and actual joint angle response $y$ of the biped robot continually optimized. Consequently, the minimized error decreases only in the range $\pm 0.5[\mathrm{deg}]$ with both of proposed AN-PID-SIG and AN-PID-HYP.

These results are really superior in comparison with the passive and unchanged error of conventional PID controller ( $\pm 2.2[\mathrm{deg}]$ in case of biped's left and right joint angle). Furthermore, in case of biped's left joint angle, Figure 6 shows that PID controller caused the biped robot joint angle response being oscillatory and unstable. On the contrary, proposed online tuning AN-PID controller continues to assert robust control to keep biped robot joint angle trajectory tracking response stable and accurate tracking.

\section{CONCLUSIONS}

An innovative online tuning adaptive neural AN-PID controller suitable for real-time robust control has been designed, developed and implemented for stable walking control of the left and right legs' joint angles of the 24-DOF full-sized biped robot in this paper. Experiment results show that the proposed online tuning adaptive AN-PID controller is able to learn the nonlinear and dynamic features of the highly nonlinear biped robot quickly and thus reduce the tracking error to nearly zero in its walking operation. The performance of the proposed neural-based AN-PID controller was found to be very good and robust in the presence of permanently external disturbances. Furthermore, with this proposed neural-based AN-PID control algorithm, both of gain scheduling value $G$ and PID parameters $K_{p}, K_{i}$ and $K_{d}$ can be modified in real time and hence actual gait trajectories can be monitored as well.
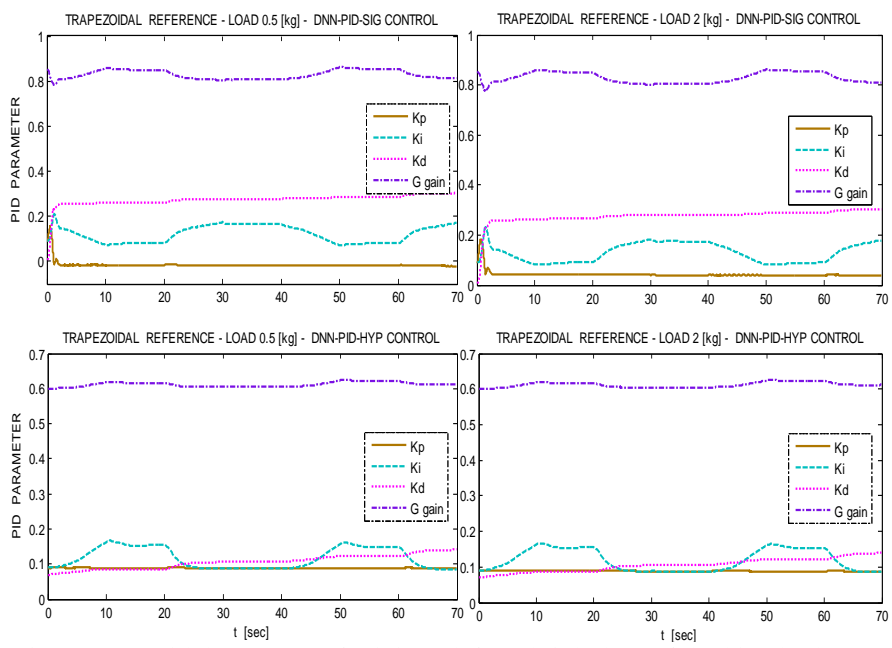

Fig.7 Trapezoidal response of 2 joints (left and right leg) of the biped robot

\section{ACKNOWLEDGEMENTS}

This research is supported by DCSELAB (funded by Vietnam National University HoChiMinh City (VNU-HCM) under grant number B2011-20b-02TĐ) and the NAFOSTED, Ha Noi, Viet Nam.

\section{REFERENCES}

[1] M. H. Raibert, Legged Robots That Balance. Cambridge, Massachusetts: The MIT Press, 1986.

[2] S. Kajita and K. Tani, "Experimental Study of Biped Dynamic Walking," in 1995 IEEE Int. Conf. on Robotics and Automation, pp. 13-19.

[3] H. Kazuo, H. Masato, H. Yuji and T. Toru, "The Development of Honda Humanoid Robot," in Conf. Rec. 1998 IEEE Int. Conf. on Robotics \&Automation, pp. 1321-1326.

[4] M. Vokobratovic, B. Borovac,D. Surla and, D. Stokic, Biped Locomotion: Dynamics, Stability, Control and Application, SpringerVerlag, 1990.

[5] Murakami, E. Yamamoto and K. Fujimoto, "Fuzzy control of dynamic biped walking robot," in Conf. Rec. 1995 IEEE Int. Conf. Fuzzy system, pp. 77-82.

[6] O. Bebek and K. Erbatur, "A fuzzy system for gait adaptation of biped walking robots," in Conf. Rec. 2003 IEEE Int. Conf. on Control Applications, pp. 669-674.

[7] D. Kim, S. J. Seo and G. T. Park, "Zero moment point trajectory modeling of a biped walking robot using an adaptive neuro-fuzzy system," IEE Proc. Control Theory Application, vol. 152, pp. A-B, July 2005.

[8] H. Wongsuwarn and D. Laowattana, "Experimental Study for FIBO Humanoid Robot," in Conf. Rec. 2006 IEEE Int. Conf. Robotics, Automation and Mechatronics (accepted).

[9] Anh, H.P.H., 2010, "Online tuning gain scheduling MIMO neural PID control of the 2-axes pneumatic artificial muscle (PAM) robot arm," Journal of Expert Systems with Applications - ESWA - Elsevier, Vol. 37, I. 9, Sep. 2010, pp. 6547-6560. 